Co porphyrins featuring electrondonating axial ligands are often competent for reduction of protons or water molecules to molecular hydrogen $\left(\mathrm{H}_{2}\right)$ (ref. 7). For the COF-based catalysts, porphyrin stacking precludes axial ligation and the materials instead tend to favour $\mathrm{CO}$ formation, typically by five- or six-fold over $\mathrm{H}_{2}$. Excessive hydrogen evolution from a solution also containing an oxygen-evolving electrode presents an explosion hazard. To the extent that hydrogen can be suppressed, electrocatalytic carbon recyclers should be safely operable without an efficiencysapping, gas-impermeable membrane. The COF-366 and COF-367 materials might well approach this ideal. Closely related work based on electrode-immobilized, metal-organic frameworks comprising metalloporphyrin linkers ${ }^{8}$ also provides an interesting comparison to these COF materials. The metal-organic framework architecture renders the candidate catalyst sites fully $\mathrm{CO}_{2}$ accessible, but at the cost of slow electron transport. The abundance of sites translates into favourable onset potentials for electrocatalysis, but slow charge transport limits the catalytic current to only a fraction of what it otherwise would be. The self-assembling COFs that the authors report provide an attractive alternative in this respect.
Idan Hod, Omar K. Farha and Joseph T. Hupp are at the Department of Chemistry, Northwestern University, Evanston, Illinois 60093, USA.

e-mail: j-hupp@northwestern.edu

\section{References}

1. IPCC Climate Change 2013: The Physical Science Basis (eds Stocker, T. F. et al.) (Cambridge Univ. Press, 2013)

2. Lin, S. et al. Science 349, 1208-1213 (2015).

3. Benson, E. E., Kubiak, C. P., Sathrum, A. J. \& Smieja, J. M. Chem. Soc. Rev. 38, 89-99 (2009).

4. Costentin, C., Robert, M. \& Saveant, J.-M. Chem. Soc. Rev. 42, 2423-2436 (2013).

5. Leung, K., Nielsen, I. M. B., Sai, N., Medforth, C. \& Shelnutt, J. A J. Phys. Chem. A 114, 10174-10184 (2010).

6. Costentin, C., Drouet, S., Robert, M. \& Saveant, J. M. Science 338, 90-94 (2012).

Kleingardner, J. G., Kandemir, B. \& Bren, K. L. J. Am. Chem. Soc. 136, 4-7 (2014)

8. Hod, I. et al. ACS Catal. 5, 6302-6309 (2015).

\title{
CORRECTING ERRORS IN SELF-ASSEMBLY
}

Symmetries seem to govern the fundamental laws of nature, but defects departures from the ideal pattern - are often what make nature interesting. Many natural patterns, from zebra stripes to sand ripples, tree branches and polygonal crack networks, acquire richness and individuality from imperfections in an underlying regularity ${ }^{1}$. Defects formed during symmetry-breaking transitions in the nascent universe might even give the cosmos itself its large-scale texture ${ }^{2,3}$.

But sometimes regularity, not variety, is what's called for, whereupon defect formation becomes a nuisance. In materials science this is arguably an ancient problem - steel tempering and annealing is ultimately concerned with defect management - and silicon-chip technology became possible thanks to single-crystal growth methods that remove grain-boundary defects. Crystallization is ultimately a form of self-assembly; but current interest in technological exploitation of more sophisticated spontaneous patterning and self-assembly of atoms and molecules has made defect manipulation a particularly pressing concern.

Such processes have the advantage of relying on simple physical laws, rather than cumbersome and slow direct manipulation or the synthetic demands incurred by programmable approaches ${ }^{4}$, to generate useful micro- and molecularscale structures. On the other hand, one is then generally restricted to the geometries that nature provides. In directed selfassembly, pattern selection may be guided by imposing boundary conditions. This idea looks particularly attractive for patterning block copolymers, which will microphase-separate to form domains whose size and morphology can be tuned via the chemistry and length of the polymer-chain segments $s^{5}$. The resulting structures might be used as resists for lithographic patterning of solids such as semiconductor films at nanometre scales, which remains challenging for conventional lithography, for example to make nanowire circuits or nanofluidic channels.

Most work here focuses on the parallel stripes and lamellae that appear in many block copolymer thin films. The regularity can be enhanced by depositing the polymers onto surfaces etched with grooves or chemically patterned with much wider stripe features. This surface patterning imposes boundary conditions that suppress more meandering, fingerprint-like morphologies ${ }^{6}$. All the same, defects do form, for example edge dislocations where one stripe jumps tracks' to an adjacent one - very much like defects seen in sand ripples.

These defects seem to be kinetically trapped structures whose thermal annealing is inhibited by a free-energy barrier ${ }^{7}$. Hur et al. have now studied them at an unprecedented level of detail using computational methods and state-ofthe-art experimental techniques ${ }^{8}$, so as to discern the most efficient pathways for defect removal and the molecular processes involved. The transition-state structures incur an enthalpic penalty due to the greater interfacial areas between immiscible polymer domains, as well as requiring conformational changes (chain stretching) of the polymer segments that bridge the dislocation to initiate 'healing' of broken stripes. This bridging

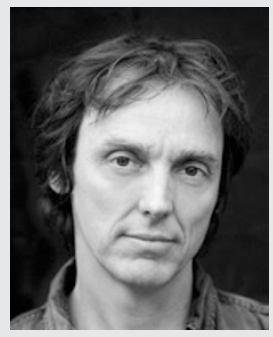

PHILIP BALL

begins at the top or bottom surfaces of the polymer film, where the energetic costs are lower.

By unravelling these details, Hur et al. can identify materials parameters that may lower the activation energy for annealing. As one might expect, this energy decreases as the incompatibility between the two blocks diminishes but so does the free energy of defects in the first place. So there is an optimum, tunable for a given copolymer composition by varying the temperature or solvent, at which thermal fluctuations may remove existing defects without creating new ones. This raises the prospect of making defect elimination more of a science than an art.

References

1. Cross, M. C. \& Hohenberg, P. C. Rev. Mod. Phys. 65, 851-1112 (1993)

2. Kibble, T. W. B. J. Phys. A: Math. Gen. 9, 1387-1398 (1976)

3. Turok, N. Phys. Rev. Lett. 63, 2625 (1989).

4. Knorowski, C. \& Travesset, A. Curr. Opin. Solid State Mater. Sci. 15, 262-270 (2011).

5. Kim, H. C., Park, S. M. \& Hinsberg, W. D. Chem. Rev. 110, 146-177 (2010).

6. Ruiz, R. et al. Science 321, 936-939 (2008).

7. Nagpal, U., Müller, M., Nealey, P. F. \& de Pablo, J. J. ACS Macro Lett. 1, 418-422 (2012).

8. Hur, S.-M. et al. Proc. Natl Acad. Sci. USA http://doi.org/8v3 (2015). 